\title{
Cardiovascular risk assessment using serum hs-CRP and Framingham risk score in newly diagnosed Graves' disease patients
}

\section{Sorina Martin1, 2, Minodora Betivoiu', Suzana Florea ${ }^{3}$, Simona Fica ${ }^{1,2}$}

1 Elias Hospital, Endocrinology Department

2 Carol Davila University of Medicine and Pharmacy, Endocrinology Department

3 Elias Hospital, Laboratory Department

Bucharest, Romania

Introduction. In hyperthyroid patients mortality is increased by $20 \%$, the major causes of death being cardiovascular disorders. Recent data suggest that subclinical or treated thyroid disease is associated with increased long-term vascular risk despite restoration of euthyroidism.

Methods. We measured high-sensitivity $C$ reactive protein (hs-CRP) in 116 newly diagnosed Graves' disease (GD) patients, without prior antithyroid treatment. Levels of hsCRP $<1 \mathrm{mg} / \mathrm{L}$ are associated with a low cardiovascular risk, between 1-3mg/L with medium risk and $>3 \mathrm{mg} / \mathrm{L}$ with high risk. We calculated Framingham risk score in 97 patients, using as risk factors: the presence of arterial hypertension, total cholesterol, HDL-cholesterol, age, sex and smoking status. Individuals with Framingham low risk score have $\leq 10 \%$ CHD risk at 10 years, with intermediate risk score $10-20 \%$, and with high risk score $\geq 20 \%$. Patients < 20 years old, with diabetes mellitus, known cardiac diseases or symptoms were excluded.

Results. The mean value of serum hs-CRP was $4.13 \pm 7.71 \mathrm{mg} / \mathrm{L}$, median=1.80, $\mathrm{IQR}=4.59 \mathrm{mg} / \mathrm{L}$. Serum hsCRP median level of $1.80 \mathrm{mg} / \mathrm{L}$ is asociated with a medium cardiovascular risk. Framingham risk score had a mean value of $2.84 \pm 3.58 \%$ SD and a median of 1 , interval=1-16, $\mid Q R=2 \%$. Framingham score placed the majority of patients (91.8\%) in a low cardiovascular risk category and only $8.2 \%$ in an intermediate risk category, with no patients in the high risk category. Framingham risk score was higher in men compared to women (mediane=4.50, $I Q R=11$ vs mediane=1, $I Q R=2 \%, p<0.001$ ). Intermediate risk patients were older than patients in the low risk group ( $64.13 \pm 10.30$ vs $43.89 \pm 13.68$ years, $p<0.001)$.

Conclusions. Serum hs-CRP values placed GD patients in a medium cardiovascular risk while Framingham risk score placed the majority of patients in a low risk group, $<10 \%$ within the next 10 years. Considering the increased morbidity and mortality associated with hyperthyroidism the development of a specific cardiovascular risk score for this specific category of patients could be useful.

Table 1. Cardiometabolic profile in 116 Graves disease patients at diagnosis

\begin{tabular}{|c|c|}
\hline Parameter & Value \\
\hline BMI, mean $\pm S D\left(k g / m^{2}\right)$ & $24.93 \pm 5.98$ \\
\hline Systolic BP, mean士SD (mmHg) & $126.29 \pm 19.21$ \\
\hline Diastolic BP, mean士SD (mmHg) & $73.32 \pm 10.54$ \\
\hline HR, mean $\pm S D($ beats/min) & $95.70 \pm 17.83$ \\
\hline Total cholesterol , meantSD(mg/dl) & $154.64 \pm 36.93$ \\
\hline HDL cholesterol, mean $\pm S D(m g / d l)$ & $50.62 \pm 14.01$ \\
\hline LDL cholesterol, mean $\pm S D$ (mg/dl) & $88.44 \pm 32.10$ \\
\hline Tryglicerides, mean士SD (mg/dl) & $101.31 \pm 46.79$ \\
\hline \multicolumn{2}{|l|}{ Smoking stałus N(\%) } \\
\hline - Never smoker & $57(46.00)$ \\
\hline - Former smoker & $23(18.50)$ \\
\hline - Active smoker & $44(35.50)$ \\
\hline Atrial fibrilation N(\%) & $13(10.50)$ \\
\hline High blood pressure N(\%) & $48(38.70)$ \\
\hline Ischemic heart disease N(\%) & $30(24.20)$ \\
\hline Diabetes mellius N(\%) & $9(7.30)$ \\
\hline - Type 1 & $2(1.60)$ \\
\hline - Type 2 & $7(5.60)$ \\
\hline
\end{tabular}

Table 2. Inflammation markers

\begin{tabular}{|c|c|}
\hline Parameter & Value \\
\hline ESR, mean $\pm S D(\mathrm{~mm} / \mathrm{h})$ & $19.22 \pm 11.87$ \\
\hline Fibrinogen, meantSD (mg/dl) & $360.26 \pm 74.95$ \\
\hline hs-CRP, median (IQR) (mg/L) & $1.80(4.59)$ \\
\hline TNFa, median (IQR) (pg/ml) & $7.0(4.90)$ \\
\hline
\end{tabular}

Table 3. Framingham risk score and cardiovascular risk

\begin{tabular}{|l|l|}
\hline Parameter & Value \\
\hline $\begin{array}{l}\text { Framingham risk score, } \\
\text { median (IQR) (\%) }\end{array}$ & $\mathbf{1}(2)$ \\
\hline Cardiovascular risk N(\%): & \\
\hline - Low & $\mathbf{8 9 ( 9 1 . 8 )}$ \\
\hline - Intermediate & $\mathbf{8 ( 8 . 2 )}$ \\
\hline - High & $\mathbf{0 ( 0 \% )}$ \\
\hline
\end{tabular}

